The published version of this manuscript is available here:

Naab, T. K. \& Schlütz, D. (2016). Nutzung von Werbung. Selektion und Vermeidung persuasiver Inhalte. In G. Siegert, W. Wirth, P. Weber \& J. Lieschka (Hrsg.). Handbuch Werbeforschung (S. 223-242). Wiesbaden: VS.

Correspondence concerning this article should be addressed to

Teresa K. Naab, Department of Media, Knowledge and Communication, University of Augsburg, Universitaetsstrasse 10, 86159 Augsburg, Germany. E-Mail: teresa.naab@phil.uni-augsburg.de, phone: +498215985933 


\title{
Nutzung von Werbung
}

\author{
Selektion und Vermeidung persuasiver Inhalte
}

\begin{abstract}
Der Kontakt mit einer Werbebotschaft ist notwendige, wenn auch nicht hinreichende Bedigung für Werbewirkung. Werbetreibende versuchen daher, die Kontaktchance ihrer Werbebotschaft durch vielfältige Strategien zu optimieren. Diesen Strategien begegnet das Publikum aktiv durch bewusste Selektion solcher persuasiver Inhalte, die es als nützlich wahrnimmt, oder aber durch Werbevermeidung (advertising avoidance). Charakteristika der Personen, die Werbung nutzen (Motive, Einstellungen, Überredungswissen, Reaktanz), aber auch der Merkmale von Werbträgern und Werbemitteln beeinflussen Nutzung und Vermeidung von Werbung. Der Beitrag diskutiert die Determinanten von Selektion und Avoidance, das Nutzungs- und Umgehungsverhalten in unterschiedlichen Medien sowie Strategien der Werbetreibenden zur Umgehung der Werbevermeidung.
\end{abstract}

\section{Stichworte zur Indexierung}

Advertising Avoidance, Attitude toward the Ad Model, Banner Blindness, Clutter, NutzungsmotivePersuasion Knowledge, Reaktanz, Uses and Gratifications Approach, Werbevermeidung, Zapping

\section{$1 \quad$ Einleitung}

Notwendige, wenn auch nicht hinreichende Bedingung für die Wirkung von Werbung auf Erinnerung, Wissen, Einstellungen und Verhalten ist ein Kontakt mit dem persuasiven Inhalt. Werbekontakt bedeutet, dem präsentierten Werbemittel (bewusst oder unbewusst) Aufmerksamkeit zu schenken und es aus der medialen Informationsfülle zu selektieren (vgl. Wirth 2001). Hierarchische Modelle der Werbewirkung stellen so auch die Nutzung einer Werbung bzw. den Werbemittelkontakt an den Anfang der Wirkungskette (Kroeber-Riel et al. 2009; McGuire 1976), Wahrnehmung gilt als "antecedent to actual processing" (Yoo et al. 2004, S. 50). Auch wenn die Verarbeitung nicht unbedingt bewusst erfolgen muss, um Spuren zu hinterlassen (vgl. z. B. mere exposure effect, Matthes et al. 2007; Zajonc 2001), haben Werbemittel, denen nur partiell Aufmerksamkeit geschenkt wird, eine deutlich geringere Wirkchance (Bellman et al. 2010). Werbetreibende streben deshalb danach, die Kontaktchance ihrer Werbemittel zu erhöhen, indem sie die Aussteuerung der Werbemittel-Auslieferung optimieren oder die Botschaften stärker in das Programm integrieren. Darüber hinaus versuchen sie, die Kontaktqualität zu verbessern, indem die Werbemittel möglichst genau auf die Zielgruppe zugeschnitten werden (Personalisierung, Targeting; Siegert und Brecheis 2010). Allerdings sind die Rezipientinnen und Rezipienten diesen Bestrebungen nicht passiv ausgeliefert, sondern können zu einem gewissen Grad steu- 
ern, welche der Werbebotschaften, die ihnen als Beiwerk anderer Medieninhalte präsentiert werden, sie selektieren und welche sie vermeiden (advertising avoidance). Mit einem Blick auf grundlegende Annahmen der Mediennutzungsforschung über die Aktivität von Rezipientinnen und Rezipienten werden deshalb im Folgenden das Nutzungsverhalten in Bezug auf Werbung sowie Determinanten der selektiven Zuwendung zu Werbebotschaften eingeordnet. Anschließend werden die Verhaltensstrategien der Nutzerinnen und Nutzer gegenüber Werbeinhalten in verschiedenen Werbeträgern beschrieben. Das Verhalten der beiden Kommunikationspartner - des aktiven, absichtsvoll selektierenden Publikums auf der einen und der eine Persuasionsabsicht verfolgenden Werbetreibenden auf der anderen Seite - bilden ein Spannungsfeld, innerhalb dessen sich Werbewirkung entfaltet.

\section{Aktive Selektion von Werbebotschaften}

Während die frühe Wirkungsforschung von einem behavioristischen Modell ausging, in dem Medienstimuli wie eine „magic bullet“ auf alle Rezipierenden treffen und gleichermaßen auf sie wirken, vollzog sich Mitte des 20. Jahrhunderts ein Paradigmenwechsel. Das aktive Publikum rückte in den Vordergrund der Betrachtung. Die Nutzerinnen und Nutzer wurden nicht mehr ausschließlich als Objekte kommunikativer Bemühungen, sondern als intentional nach ihren Bedürfnissen handelnde Subjekte verstanden, die sich Kommunikationsinhalten absichtsvoll zuwenden - oder, wie gelegentlich im Fall von Werbung, auch bewusst davon abwenden. Aktiv Rezipierende wählen nicht-zufällig aus dem medialen Angebot und anderen, nicht-medialen Alternativen aus (Selektivität). Ihre Auswahl wird von Wissen und Absichten geleitet (Intentionalität) und führt sie zu Angeboten, von denen sie die Befriedigung von Bedürfnissen erwarten (Nützlichkeit). Involvierte Rezipierende stellen eine Verbindung zwischen sich und dem Kommunikationsinhalt her und verarbeiten die Botschaften unter Aufwendung kognitiver Ressourcen (zum Begriff der Aktivität ausführlicher z. B. Biocca 1988; Blumler 1979; Levy und Windahl 1985). Die Konzeption des Publikums als aktiv konkretisierte sich im Uses-and-Gratifications-Ansatz. Katz et al. (1974) fassen dessen Grundannahmen zusammen: Das Medienpublikum nutzt die Medien zum Erreichen von Zielen. Allerdings sind die Medien nicht die einzige Quelle der Bedürfnisbefriedigung, sondern sie konkurrieren mit nicht-medialen Aktivitäten. Zwischen diesen Alternativen wägen die Menschen ab. Die subjektiven Motive der Mediennutzerinnen und -nutzer sind der Schlüssel zur Erklärung der Rezeption. Grundannahme des Ansatzes ist, dass sich Menschen ihrer Motive für die Mediennutzung bewusst sind und dass sie darüber Auskunft geben können (z. B. Greenberg 1974; LaRose und Eastin 2004; Rubin 1984). Auch in der Werbeforschung vollzog sich die beschriebene Wende hin zum aktiven Publikum. Während die Rezipientinnen und Rezipienten zuvor als weitgehend passive Empfänger von Werbebotschaften gesehen wurden, rückte ihr aktiver Umgang mit Werbung in den Vordergrund der Betrachtung (O'Donohoe 1994). Menschen, die aktiv Werbung rezipieren, können beeinflussen, welche Werbebotschaften sie nutzen oder vermeiden. Ihre Motive werden zu entscheidenden Determinanten der Werbenutzung und damit auch für eine mögliche spätere Wirkung. In der Weiterentwicklung erhielt der Ansatz eine stärkere theoretische Fundierung. Unter anderem wurden die in einem Medienangebot bei der Nutzung gesuchten Gratifikationen von den tatsächlich erhaltenen unterschieden (Palmgren und Rayburn 1982, 1985). Dabei ist allerdings zu berïcksichtigen, dass sich die Gratifikationen (zumindest in klassischen Befragungen) häufig eher auf Medienimages, denn auf konkrete Medieninhalte beziehen (Scherer und Schlütz 2002). Mit Blick auf Werbung erfassen solche Befragungen dann häufig allgemeine Motive der Werbenutzung, unabhängig vom Werbeträger, dem Werbemittel, der konkreten Botschaft oder der spezifischen Mediennutzungssituation oder sie erfassen - noch allgemeiner - generelle Einstellungen gegenüber Werbung. Es ist unmittelbar einleuchtend, dass sich dadurch be- 
stimmte Unschärfen ergeben, da verschiedene Ausgestaltungen einer Botschaft in Form eines Werbemittels in einem spezifischen Werbeträger sehr unterschiedliche Gratifikationen erfüllen können. So mag ein Viralspot Unterhaltungsbedürfnisse befriedigen, eine sog. „Schweinebauch“-Anzeige aber ihres Informationsgehaltes wegen genutzt werden. Pauschale Befragungen werden diesen Besonderheiten einzelner Botschaften nicht ohne weiteres gerecht.

\section{Determinanten der Zuwendung zu Werbung}

Selektion oder Vermeidung von Werbebotschaften hängt von verschiedenen Faktoren ab. Neben Nutzungsmotiven determinieren die allgemeine Einstellung gegenüber Werbung und Persuasionsversuchen die Zuwendung. Aber auch das Involvement des Rezipienten oder der Rezipientin spielt eine Rolle. Je stärker etwa das Programminvolvement ausgeprägt ist, umso größer ist die Wahrscheinlichkeit, dass eine Werbebotschaft beachtet wird (Moorman et al. 2007). Bei geringerem Involvement werden andere Einstellungsvariablen wichtiger. Auf diese gehen wir im Folgenden ein.

\subsection{Nutzungsmotive}

In qualitativen wie quantitativen Befragungen wurden konkrete Motive für die Zuwendung zu Werbeinhalten, erhaltene Gratifikationen aus der Nutzung konkreter Werbebotschaften in verschiedenen Medien sowie allgemeine Einstellungen gegenüber Werbung ermittelt. Dabei haben sich folgende Dimensionen der Nutzungsmotivation wiederholt gezeigt (Alwitt und Prabhaker 1992; Mitchell et al. 2007; O'Donohoe 1994; Plummer 1971; Schlinger 1979):

- Informationsmotiv: Die Rezipientinnen und Rezipienten nutzen Werbung für Informationen über Produktmerkmale und Produktneuheiten, als Hilfestellung für Kaufentscheidungen sowie deren nachträgliche Absicherung. Gerade bei Kaufentscheidungen mit einem hohen wahrgenommenen Risiko und hohem Involvement in den Kauf er-

folgt eine umfangreichere Informationssuche. Insbesondere bei weniger bekannten, seltener gekauften und höherpreisigen Produkten oder Produkten mit vermeintlich sozialem Risiko informieren sich die Konsumentinnen und Konsumenten mithilfe von Werbung (Bucklin 1965; Kroeber-Riel et al. 2009). Ein ausgeprägtes Informationsbedürfnis fördert Selektion und Nutzung relevanter Werbeinhalte. Darüber hinaus können Werbebotschaften Informationsbedürfnisse erfüllen, die jenseits der Kaufbeeinflussung liegen, indem sie den Rezipientinnen und Rezipienten Bildung über gesellschaftlich relevante Themen vermitteln.

- Soziale Motive: Die Rezipientinnen und Rezipienten halten Werbung für nützlich in der sozialen Interaktion mit Familie und Freunden. Beispielsweise dienen neue und bekannte Werbeinhalte als Gesprächsstoff, bei dem man mitreden können möchte. Werbeslogans finden Eingang in die Alltagssprache und unterstützen die Kohäsion in einer Gruppe durch die wiederholte gemeinsame Verwendung. Werbung kann darüber hinaus Bedürfnisse nach Identitätsbildung befriedigen, indem sie hilft, den eigenen Lebensstil oder Werthaltungen zu bestätigen, einen Aufwärtsvergleich anzustellen, indem sie Rollenmodelle präsentiert oder zu Empathie anregt. Umgekehrt spiegelt die Werbung den „Distinktionswert“ von Konsumgütern und Marken (Hellmann 2006: 398) und kann auf diese Weise helfen, andere Konsumentinnen und Konsumenten einzuordnen. Auch diese Bedürfnisse fördern die Zuwendung zu persuasiven Inhalten.

- Unterhaltungsmotiv: Schließlich suchen Nutzerinnen und Nutzer nach Unterhaltung und Stimulation. Sie regen ihre Fantasie durch Werbeinhalte an, nutzen sie als kreativen Input, um sie sprachlich, gestalterisch oder durch Nachspielen kreativ zu verarbeiten. Teilweise werden Werbeinhalte als mindestens so unterhaltsam wie das eigentliche 
Medienangebot eingestuft (O'Donohoe 1994). Schließlich suchen Personen Zerstreuung oder nutzen Werbeangebote aus eskapistischen Motiven und zur Strukturierung ihrer Mediennutzungszeit durch Werbeunterbrechungen.

Rezipientinnen und Rezipienten werden aber auch mit unerwünschter Werbung konfrontiert, die nicht ihren durch die Mediennutzung angestrebten Gratifikationen entspricht. Werbung kann dann unter Umständen den Kommunikationsfluss stören, indem sie die Suche der Nutzerinnen und Nutzer nach nicht-werblichem Medieninhalt behindert (search hinderance), dessen Verarbeitung stört (distraction) oder sogar unterbricht (disruption; Speck und Elliott 1997). Sie behindert damit ggf. die eigentlich mit Mediennutzung verfolgten Ziele der Rezipierenden (perceived goal impediment; Cho und Cheon 2004) und wird als aufdringlich wahrgenommen (intrusiveness; Li et al. 2002). Findet eine Rezipientin oder ein Rezipient keinen Mehrwert in einem Werbeangebot und stört dieses bei der Erreichung der eigentlichen Ziele der Mediennutzung, löst die Werbung Irritation aus (Ducofee 1995, 1996; Edwards et al. 2002; Pasadeos 1990). Dies kann, sogar bei nicht bewusst verarbeiteten Werbemitteln, zu negativer Bewertung derselben führen (distractor devaluation; Duff und Faber 2011). Die allgemeine Mediennutzungsmotivation spielt also eine Rolle für die Wahrnehmung von Werbung. Werbung wird z. B. unterschiedlich wahrgenommen, in Abhängigkeit davon, ob die Rezipierenden eine bestimmte Absicht mit der Mediennutzung verfolgen (goal/task orientation) oder weniger zielgerichtet sind, z. B. ohne Ziel surfen (exploratory, playful). Eine stärkere Zielorientierung wirkt sich zumeist negativ auf die Werbewahrnehmung aus (Zanjani et al. 2011; Danaher und Mullarkey 2003; Duff und Faber 2011; Klimmt et al. 2009). Je stärker Werbung die Suche nach nicht-werblichem Inhalt behindert, desto umfassender ist die Werbevermeidung. Auch der Umfang der medialen Alternativen trägt zum Ausmaß der Werbevermeidung bei, was den aktiven Charakter der Werbenutzung unterstreicht (Cho und Cheon 2004; Speck und Elliott 1997). Gerade Werbung mit vermeintlich unwahrem, beleidigendem oder verwirrendem Inhalt, mit schlechter Umsetzung und Gestaltung und zu häufiger Platzierung mit zu vielen anderen Botschaften (clutter), wirkt irritierend und wird vermieden (Bauer und Greyser 1968; Cho und Cheon 2004). Die Form der Störung sowie der Umgang damit sind abhängig vom Werbeträger und der Art der Werbung. Weiter unten gehen wir deshalb auf Formen der Werbevermeidung in verschiedenen Medien ein.

\subsection{Einstellung gegenüber Werbung}

Das Attitude toward the Ad Model (vgl. MacKenzie et al. 1983; MacKenzie und Lutz 1989) formuliert, dass die allgemeine Einstellung gegenüber Werbung Einfluss auf die Einstellung zu einem bestimmten Werbemittel hat (und damit auf die daraus erwachsenden Konsequenzen). Es finden sich Hinweise in der Literatur, dass Werbekontakt oder -vermeidung nicht ausschließlich eine Reaktion auf bestimmte Werbebotschaften, ihre Umsetzung und Platzierung ist, sondern auch von einer allgemeinen Einstellung gegenüber Werbung (also einer erlernten Disposition, die von Akzeptanz bis Skepsis reichen kann) determiniert wird. Einstellungen sind relevant, weil sie zwischen Einstellungsobjekt (z. B. Werbung im Allgemeinen) und Verhalten (z. B. Beachtung, Produktkauf) vermitteln. So wirkt sich eine positive Einstellung gegenüber Werbung vorteilhaft auf die Beachtung aus (Winkler und Buckner 2006). Aber auch der gegenteilige Effekt ist belegt: Manche Rezipientinnen und Rezipienten vermeiden nicht nur einzelne Werbeinhalte, sondern wenden sich grundsätzlich ab, sobald sie Werbung ausgesetzt werden (Li et al. 2002). Speck und Elliott (1997) können entsprechend in einer Studie zeigen, dass die Einstellung gegenüber Fernsehwerbung im Vergleich zur Einstellung gegenüber Zeitungs-, Zeitschriften und Radiowerbung am negativsten ist, weshalb Fernsehwerbung die höchsten Vermeidungsraten hat (hierzu auch Rojaz-Méndez und Davis 2005; Tan und Chia 2007; vgl. zur Messung von Werbeeinstellungen den Beitrag von Schlütz im vorliegenden Band). 
Grundsätzlich lassen sich zwei Einstellungsbereiche voneinander trennen: der eher makroperspektivisch angelegte ökonomische oder gesellschaftliche Nutzen von Werbung (z. B. auch zur Finanzierung von Medien, siehe auch den Beitrag von Seufert und Lischka im vorliegenden Band) und der individuelle Nutzen (etwa in Form von Information oder Unterhaltung) bzw. die Abwesenheit desselben (i. S. v. Werbeskepzis, vgl. Obermiller und Spangenberg 1998). Pollay und Mittal (1993) differenzieren entsprechend zwischen Einstellungen gegenüber gesellschaftlichen und persönlichen Auswirkungen von Werbung. Im Hinblick auf gesellschaftliche Effekte trennen sie Einstellungen zu den wirtschaftlichen Benefits durch Werbung, zu Auswirkungen auf die materialistische Grundhaltung der Gesellschaft, zum Beitrag von Werbung zum Werteverfall und zum Wahrheitsgehalt von Werbung (siehe dazu auch die Beiträge von Zurstiege und Haas/Herczeg/Karsay in diesem Band). Die Einstellungen zu den persönlichen Effekten von Werbung entsprechen im Wesentlichen individuellen Motiven für die Werbenutzung, nämlich Information, Unterhaltung und sozialem Statusstreben (siehe auch Alwitt und Prabhaker 1992; Kwak et al. 2009; Speck und Elliott 1997; Tsao und Sibley 2004). Repräsentative Studien zur Einstellung der Deutschen zeigen eine hohe Akzeptanz von Werbung. Sie sehen vor allem ihre wirtschaftliche Bedeutung, empfinden Werbung als einen normalen Teil des modernen Lebens und erkennen ihren Informationswert an. Auch Fernsehwerbung im Speziellen wird akzeptiert. Allerdings wird Unterbrecher-Werbung innerhalb von Sendungen abgelehnt (Ridder und Hofsümmer 2001, 2008).

\section{3 Überredungswissen und Reaktanz}

Mediennutzerinnen und -nutzer wählen nicht nur zielgerichtet Inhalte aus und versuchen durch ihre Abwendung von Werbeinhalten Werbekontakte zu umgehen, sondern sie wissen auch grundsätzlich um die kommerzielle und persuasive Absicht von Werbung. Mit dem Erkennen der Werbeintention geht häufig ein kognitiver Abwehrmechanismus einher, der die Nutzung einschränkt oder verhindert und der der Wirkung abträglich sein kann. Dieses Überredungswissen (persuasion knowledge; Friestadt und Wright 1994, 1995) ist abhängig von persönlichen Erfahrungen mit Überzeugungsversuchen und variiert deshalb zwischen Personen. Es kann Wissen über die psychologischen Prozesse beinhalten, die Werbende zu beeinflussen versuchen (z. B. Aufmerksamkeit, Vorstellungen, Gefühle, Absichten), die Ziele und Taktiken, die die Werbetreibenden verwenden, die wahrgenommene Effektivität und Angemessenheit sowie die eigenen Bewältigungsstrategien im Umgang mit den Überredungsversuchen. Die Wahrnehmung eines Überredungsversuchs kann bei Rezipierenden Reaktanz erzeugen. Reaktanz entsteht als Abwehrreaktion auf die vermeintliche Einschränkung von Freiheit. Indem sich Betroffene dem wahrgenommenen Druck widersetzen, versuchen sie ihre Freiheit wiederherzustellen (Brehm 1966). Das gilt auch für wahrgenommene Überredungsversuche durch Werbung (Schlütz und Hartmann 2012). Nehmen Rezipierende einen Überredungsversuch wahr, d. h. wird ihr Überredungswissen aktiviert, verarbeiten sie die Werbebotschaft vor diesem Hintergrund. Das Überredungswissen kann den Kontakt, aber auch die Wirkung der Werbekommunikation moderieren (Friestad und Wright 1994, 1995). Beispielsweise kann unter bestimmten Bedingungen hohes Überredungswissen zu einer negativeren Markenwahrnehmung beitragen (Cowley und Barron 2008; Matthes et al. 2007) und die wahrgenommene Glaubwürdigkeit von Botschaften reduzieren (Wood et al. 2008).

\section{$4 \quad$ Werbenutzung und -vermeidung}

Den Rezipierenden kommt eine zunehmend aktive Rolle im Prozess der Werbenutzung zu; ihr Verhalten wird verstärkt selektiv (vgl. Baek und Morimoto 2012; Cho und Cheon 2004; Pagendarm und Schaumburg 2001; Pavlou und 
Stewart 2000). In Abhängigkeit von den in Werbeinhalten gesuchten und erhalten Gratifikationen, der Bewertung einer speziellen Werbebotschaft sowie den individuellen Einstellungen einer Person gegenüber Werbung im Allgemeinen und deren persuasiven Strategien, begegnen Rezipientinnen und Rezipienten dem kommunikativen Angebot in unterschiedlicher Weise. Sie suchen aktiv nach Werbebotschaften, rezipieren die Werbeinhalte, die ihnen im Kontext eines anderen Programms präsentiert werden oder sie wenden sich von den präsentierten Werbebotschaften ab.

\subsection{Messung von Werbenutzung und -vermeidung}

Methodisch gehen Forscherinnen und Forscher Untersuchungen zur Werbenutzung und -vermeidung durch retrospektive Selbst-Berichte, Tagebucherhebungen, Beobachtungen im heimischen Umfeld der Probandinnen und Probanden und im Labor und mit Hilfe telemetrischer Daten an. Entsprechend der angewendeten Methoden variieren die Ergebnisse über die Nutzungs- und Avoidance-Raten. Hinzu kommen außerdem Unterschiede in der Stichprobenziehung, dem Erhebungsjahr, den Untersuchungsländern und den jeweils konkret betrachteten Formen der Werbevermeidung. Sie erschweren Beurteilung und Vergleich der Ergebnisse (im Überblick Bellmann et al. 2010; Dix und Phau 2010).

Studien, in denen die Befragten selbst retrospektiv Auskunft über ihre Werbenutzung und ihre Umschaltverhalten geben (z. B. Heeter und Greenberg 1985) sind mit Verzerrungen durch Erinnerungs- und Schätzfehler und sozial erwünschten Antworttendenzen behaftet. Diese Fehler lassen sich durch zeitnahe Abfragen abmildern. Tse und Lee (2001) befragen beispielsweise Fernsehnutzerinnen und -nutzer in Telefoninterviews direkt im Anschluss an Werbeunterbrechungen auf bekannten Fernsehsendern in der Prime Time. In ähnlicher Weise versuchen Forschende durch Tagebucherhebungen die Verzerrungen, die durch retrospektive Erfassung entstehen, zu mildern. Allerdings sind diese mit großem Aufwand für die Probandinnen und Probanden verbunden und meist nur eingeschränkt repräsentativ (zur Methode vgl. Naab 2013).

In Beobachtungsstudien notieren anwesende Kodiererinnen und Kodierer den Umfang der Programm- und Werbenutzung, die Umschalthäufigkeit, die Nebentätigkeiten oder die eyes-on-screen-Zeit (z. B. Krugman et al. 1995). Alternativ werden die genannten Daten mit Video- oder Tongeräten aufgezeichnet (z. B. Cronin und Menelly 1992). Beobachtungsstudien sind meist auf kleine, nicht repräsentative Stichproben beschränkt und die beobachteten Mediennutzerinnen und -nutzer könnten ihr Verhalten ob der besonderen Situation anpassen (zur Methode vgl. Gehrau und Schulze 2013). Die telemetrische Messung des Fernsehverhaltens ist eine Form der apparativen Beobachtung. In Deutschland werden die Fernsehreichweiten von der GfK-Fernsehforschung im Auftrag der Arbeitsgemeinschaft Fernsehforschung (o. J.) repräsentativ für die deutsche Wohnbevölkerung erhoben. Das Unternehmen verwendet People-Meter-Geräte, bei denen jede fernsehende Person sich durch einen Knopf auf einer Fernbedienung bei jeder Nutzung registriert. Die technische Beobachtung hat den Vorteil, dass Zuschauende kaum in ihrem Verhalten gestört werden und nicht selbst Auskunft geben müssen. Forschende sind hier nicht auf die Auskunftsfähigkeiten der Teilnehmenden angewiesen; Verzerrungen durch Erinnerungsfehler werden vermieden (zur Methode vgl. Ettenhuber 2007). Mittlerweile können auch die zeitversetzte Nutzung mit digitalen Festplattenrekordern und auch das Überspringen von Werbung durch Vorspulen telemetrisch erfasst werden (Nielsen 2007; TiVo 2006).

Auch der Kontakt mit Online-Werbung wird häufig apparativ erfasst. Gemessen werden Verhaltensindikatoren wie Klickrate (click through rate, CTR; d. h. der Anteil der Klicks auf das Werbemittel an den gesamten Impressions) oder Conversion Rate (der Anteil der Personen, deren Werbekontakt in einen Kauf verwandelt wurde). Der Vorteil dieser Indikatoren besteht darin, dass sie harte Werbewirkungen in Echtzeit erfassen (was allerdings nicht die einzig relevanten Faktoren sind; vgl. Fulgoni und Mörn 2009). 
Die Nutzung von stärker in das Programm integrierten Werbeformen wie Product Placement, spezifischen Formen der Online-Werbung oder sog. In-Game Advertising (Werbung in Bildschirmspielen; siehe unten) wird ähnlich erhoben, häufig aber im Rahmen von Beobachtungs- oder Befragungsexperimenten (z. B. Blake et al. 2012; Schlütz et al. 2013).

\subsection{Publikumsstrategien zur Nutzung und Vermeidung von Werbekontakten}

Um Werbung zu umgehen, wenden Rezipientinnen und Rezipienten verschiedene Strategien an (Speck und Elliott 1997). Sie können die Werbung ignorieren und ihre Aufmerksamkeit auf Tätigkeiten parallel zur Mediennutzung richten (cognitive avoidance). Sie können sich abwenden, den Raum verlassen oder die Printmedienseite mit der Werbebotschaft umblättern (Handlungsstrategie zur Vermeidung). Letztlich können sie das Programm wechseln (mechanical avoidance; vgl. auch Kelly et al. 2010). Neben kognitive Selektionsprozesse und etablierte Werbevermeidungsstrategien wie Umschalten oder Überblättern von Werbung treten technische Hilfsmittel zur Filterung von Umweltreizen wie beispielsweise Adblocker oder digitale Videorekorder (Wilbur 2008).

Möchten Rezipierende Werbung vermeiden, haben sie unterschiedliche Möglichkeiten abhängig von der Struktur des genutzen Medienangebots, weil dieses den Handlungsspielraum beeinflusst. Das Fernseh- und Radioprogramm folgt einem linearen, zeitlich strukturierenten Ablauf, an den die Rezipierenden gebunden sind. Dieser wird von Werbeeinspielungen unterbrochen. Die Rezipientinnen und Rezipienten können diese Werbebotschaften umgehen, indem sie den Raum verlassen oder ihre Aufmerksamkeit auf andere Tätigkeiten richten bis der Werbeblock vorbei ist. Möchten sie die Rezeption fortsetzen, bleibt ihnen, das Programm zu wechseln (zapping, channel switching). Steht Technik zur zeitunabhängigen Nutzung zur Verfügung, wie ein Video- oder digitaler Festplattenrekoder oder die Nutzung über eine Mediathek, können die Zuschauerinnen und Zuschauer den linearen Ablauf umgehen, indem sie die Werbeeinblendungen durch Vorspulen überspringen (zipping; Speck und Elliott 1997). Auch in Printmedien unterbrechen Werbeanzeigen den nicht-kommerziellen Inhalt optisch und behindern ein übergangsloses Weiterlesen. Allerdings können die Leserinnen und Leser die Dauer selbst bestimmen, die sie auf die Werbeanzeigen verwenden möchten, und ggf. umgehend weiterblättern oder ihre Aufmerksamkeit auf andere Teile der Seite richten. Klassische Online-Werbeformen (Display Advertising wie Banner oder Skyscraper) werden je nach Lage auf der Website übersehen (Banner Blindness) oder müssen aktiv vermieden werden (etwa durch das Wegklicken von Pop Ups oder Layer Ads, die den eigentlichen Seiteninhalt überlagern). Stärker in das Programmumfeld integrierte Werbeformen wie Product Placement versprechen eine höhere Kontaktchance, weil sie nicht umgangen werden können, ohne die eigentliche Nutzung abzubrechen.

Ob der bereits beschriebenen methodischen Schwierigkeiten und der Unterschiede zwischen den Studien, sind Aussagen über den tatsächlichen Umfang der Werbenutzung bzw. -vermeidung fehlerbehaftet. Hinzu kommt, dass die verschiedenen Mediengattungen kaum gleichermaßen untersucht sind. Ein Fokus liegt auf Studien zur Vermeidung von Fernsehwerbung. Als Richtung lässt sich festhalten: Zwar schalten Zuschauende auch während des Fernseh- und Radioprogramms um, Umschaltvorgänge während Werbeunterbrechungen sind allerdings signifikant häufiger (für Fernsehen siehe Moriaty und Everett 1994; für Radio siehe Heeter und Cohen 1988). Dennoch zeichnen Untersuchungen zur mechanischen Werbevermeidung ein für Werbetreibende eher beruhigendes Bild. Bellmann et al. (2010) kommen in einer Zusammenschau diverser Studien unterschiedlicher Methodik zur Vermeidung von Fernsehwerbung zu dem vorsichtigen Ergebnis, dass mechanische Werbevermeidung durch Umschalten oder Vorspulen etwa 10 Prozent der Fernsehwerbung betrifft. Auch für den deutschen Markt zeigen Hofsümmer und Müller (1999), dass durchschnittlich 
80 Prozent der deutschen Zuschauerinnen und Zuschauer während eines Werbeblocks dem eingeschalteten Programm treu bleiben. Außerdem werden diese Verluste teilweise kompensiert durch Nutzerinnen und Nutzer, die während des Werbeblocks neu auf das Programm stoßen.

Je höher der wahrgenommene Wert einer Werbung ist, desto geringer ist die Wahrscheinlichkeit, dass die Werbebotschaft weggezappt wird: Fernsehwerbung für Produkte, die der Befragte häufiger kauft, wird weniger häufig gemieden, ebenso wie Werbung, die relevante Informationen über das beworbene Produkt gibt. Werbespots, die Befragte bereits kennen, aber nicht zu häufig gesehen haben, werden seltener weggeschaltet (Siddarth und Chattopadhyay 1998). Dies unterstreicht die Aktivität der Nutzerinnen und Nutzer, die sich Werbeinhalten zielgerichtet zur Erfüllung ihrer Bedürfnisse zuwenden. Darüberhinaus beeinflussen auch Merkmale der Rezipierenden (Ottler 1998; Speck und Elliott 1997) sowie die Platzierung der Werbebotschaft Nutzung bzw. Vermeidung. Tendenziell sind die Fernsehzuschauendenbewegungen bei langen Werbeblöcken, in der Prime-Time und bei längeren Programmen größer (Hofsümmer und Müller 1999; van Meurs 1998; Zufryden et al. 1993; einen Hinweis auf geringere Vermeidungsraten bei vielen Spots finden allerdings Danaher 1995 sowie Hammer et al. 2009). Werbungen an frühen Positionen werden eher weggezappt (für Radio Abernethy 1991).

Wird ein Medieninhalt nicht im Live-Programm verfolgt, ist es möglich die Werbeunterbrechung durch Vorspulen (zipping) zu überspringen. Die Raten für durch Zipping übersprungene Werbebotschaften sind tendenziell höher als für die durch Umschalten auf einen anderen Sender verpasste. Meist werden ganze Werbeblöcke und Werbungen am Ende eines Programms vorgespult (Cronin und Menelly 1992; Downey 2007), was die Wahrnehmung der Werbebotschaften allerdings nicht komplett verhindert. Im Gegenteil gibt es Hinweise, dass vorgespulte Werbung mehr Aufmerksamkeit erhält, weil die Nutzenden sich konzentrieren, den Wiederbeginn des Programms nicht zu verpassen (z. B. Stipp 2008). Auch Werbung, die nur teilweise gesehen wird, weil die Person den Raum verlässt oder den Ton abschaltet, zeigt Effekte (Bellmann et al. 2010; im Überblick Wilbur 2008).

Unterbrecher-Werbung in linear strukturierten Programmabläufen muss nicht zwangsläufig durch die Suche nach (vorübergehenden) Programmalternativen umgangen werden, sondern kann auch - bei meist weiterlaufendem Programm - physisch oder kognitiv gemieden werden. Nebenbeitätigkeiten während der Mediennutzung sind keinesfalls auf die Werbezeiten beschränkt. Gerade Radio gilt als das klassische Nebenbeimedium (z. B. Kuhlman und Wolling 2004 zu Paralleltätigkeiten während des Fernsehens; Gattringer und Klingler 2013 während der Radionutzung). Allerdings zeigt sich, dass während Werbeeinblendungen die Aufmerksamkeit signifikant häufiger auf andere Tätigkeiten gelenkt wird als während des Fernsehprogramms (Krugman et al. 1995; Moriaty und Everett 1994). Bellmann et al. (2010) beziffern den Umfang der physical avoidance von Fernsehwerbung auf etwa 20 Prozent und den der kognitiven Vermeidung auf etwa 40 Prozent.

\subsection{Strategien der Werbetreibenden zur Umgehung der Werbevermeidung}

Das aktive Publikum nutzt Werbung zielgerichtet, umgeht sie aber auch häufig. Durch eine Optimierung der Kontakthäufigkeit, der besonderen Platzierung innerhalb von Werbeblöcken, der Optimierung der Länge von Werbeblöcken sowie durch die Verwendung von Sonderwerbeformen, versuchen die Werbetreibenden die Zuschauerabwanderung zu verhindern. Darüber hinaus haben werbetreibende Unternehmen und Werbeträger neue Werbeformen entwickelt, um diesem Problem zu begegnen (siehe dazu u.a. den Beitrag von von Rimscha/Siegert sowie Gleich im vorliegenden Band). Die Formen verschmelzen Pogramm und Werbung auf eine Art und Weise, die Werbevermeidung erschwert oder unmöglich macht, ohne auf Teile des nicht-kommerziellen Medieninhalts verzichten zu müssen. Solche 
integrierten oder hybriden Werbeformen umgehen die klassische Trennung von Programm und Werbung (Siegert und Brecheis 2010). In filmischen Medien (Fernsehen, Kino, aber auch Bildschirmspiele) erfolgt die Integration von Werbung und Programm vielfach in Form von Product Placement (vgl. hierzu den Beitrag von Koch im vorliegenden Band). Diese Werbeform hat den Vorteil, dass sie häufig gar nicht als Werbung wahrgenommen (DeLorme und Reid 1999) und daher nicht aktiv vermieden wird. Falls doch, kann man sie nicht umgehen, ohne auch auf Teile des Programmes verzichten zu müssen. Der Werbekontakt erfolgt hier nebenbei und wird häufig nicht als störend empfunden (DeLorme und Reid 1999).

Eine andere Möglichkeit der Werbeintegration ist es, Werbung parallel zum Programminhalt anzubieten wie etwa bei Split-Screen-Werbeformaten im Fernsehen oder in Form von Online-Bannern oder Ad Words auf Websites. Studien zeigen allerdings, dass herkömmliche Online-Werbemittel häufig kaum noch wahrgenommen, also passiv vermieden werden (Banner Blindness, vgl. Drèze und Hussherr 2003; Klimmt et al. 2009; Pagendarm und Schaumburg 2001). Obwohl die Anzeigen im Gesichtsfeld ausgespielt werden, werden sie nicht bewusst wahrgenommen und entsprechend auch nicht erinnert. Eine Alternative für Werbetreibende ist es daher, auffälligere Werbemittel zu nutzen wie z. B. Pop Up Ads (Edwards et al. 2002). Da sich das Werbemittel hier über den eigentlichen Medieninhalt legt, entsteht auf jeden Fall ein Werbemittelkontakt. Dieser dauert zumeist auch länger als ein Kontakt mit Werbemitteln, die im Randbereich platziert werden (Blake et al. 2012; Lee und Faber 2007). Allerdings führt das nicht unbedingt zu einer besseren Erinnerung (Diao und Sundar 2004). Darüber hinaus kann sich ein solcher erzwungener Kontakt auch negativ auf die Einstellung zum Werbemittel oder zur Marke auswirken (Burns und Lutz 2006), weil die User sich gestört fühlen und Reaktanz entwickeln (Duff und Faber 2011).

Anders als andere Werbeformen erzeugt Product Placement in Bildschirmspielen (sog. In-Game Advertising) kaum Reaktanz, da viele Spielerinnen und Spieler die Werbung akzeptieren, wenn sie sich einpasst (Lewis und Porter 2010; Wise et al. 2008) und zu einer realistischeren bzw. attraktiveren Gestaltung der Spielumwelt beiträgt (vgl. Nelson 2005; Nelson et al. 2004). Die Integration der Werbung ins Spiel ist dabei unterschiedlich stark ausgeprägt. Sie reicht von Around Game Advertising (also klassischer Online-Werbung, die im Umfeld eines Browser-Games platziert ist), über eine Individualisierung der Spielumgebung (customization; vgl. Dardis et al. 2012) bzw. den Einsatz von Branded Virtual Goods in Social Games bis hin zu Advergames, also Spielen, die speziell entwickelt wurden, um für die Marke bzw. das Produkt eines Herstellers zu werben. Hier sind Werbemittel und Medieninhalt komplett verschmolzen.

\section{Ausblick}

Das aktive Publikum nutzt Werbung gezielt, wenn sie bedürfnisgerecht ist. Es vermeidet sie (aktiv oder passiv), wenn dem nicht so ist. Das Selektionsverhalten nimmt mit der Allgegenwart von Werbung zu. Wie Mediennutzerinnen und -nutzer ihre Strategien für Selektion und Vermeidung von Werbung weiterentwickeln, so verfeinern Werbetreibende ihre Verfahrensweisen, um die Kontaktchance der Werbung zu verbessern. Mediapläne werden dahingehend optimiert, dass Kontakthäufigkeit und spezifische Werbeträgerkombinationen (Zigmond und Stipp 2010) ideale Ergebnisse erzielen. Darüber hinaus geht der Trend hin zu personalisierten, intergrierten, interaktiven Werbeformen, die Streuverluste vermeiden, Werbevermeidung umgehen und eine hochinvolvierte Nutzung induzieren.

Den Nutzungs- bzw. Vermeidungsmustern begegnen Werbetreibende darüber hinaus, indem sie versuchen, Nutzungsfrequenz und Kontaktqualität einzelner Werbemittel zu verbessern. Sie bedienen sich dafür z. B. besonders salienter Werbemittel (Simola et al. 2011), solchen, die sich kaum umgehen lassen (z. B. Pop Ups oder Layer Ads), integrieren 
verstärkt Werbung und Programm (z. B. Product Placement, Advertorials im Printbereich oder die Online-Variante, das sog. Native Advertising), nutzen innovative Below-the-Line-Werbeformen (wie z. B. Ambient Media Kampagnen oder Guerilla-Marketing-Aktionen mit anschließender viraler Verbreitung), persönliche Empfehlungen (Word of Mouth Marketing) oder sprechen Verbraucherinnen und Verbraucher im öffentlichen Raum an (mobile Werbung; siehe auch den Beitrag von Gleich zu Sonderwerbeformen und Fahr/Bell zu Online- und mobiler Werbung im vorliegenden Band und Weber, Buchmann und Wirth 2014 zu Ambient Werbung).

Eine weitere Möglichkeit, Aufmerksamkeit und Nutzung anzuregen, ist, interaktive Werbeformen einzusetzen. Diese fördern spielerisch das Engagement mit der Marke und erhöhen dadurch die Kontaktqualität mit dem Werbemittel. Solche Strategien werden vor allem bei (interaktiven) Bildschirmspielen eingesetzt. Sie ermöglichen eine weiterreichende Art der Auseinandersetzung mit den beworbenen Marken oder Produkten, da die Spielenden kognitiv (und z.T. auch emotional; vgl. Nicovich 2005) involviert werden. Dabei ist durchgängig ist ein Trend zur sog. Gamification zu beobachten (vgl. Terlutter und Capella 2013). Damit wird ein Prozess bezeichnet, der Merkmale des Spiels (sog. game mechanics wie achievement, competition, sharing) auf andere Bereiche wie z. B. Werbung anwendet. Solche ,pparticipation-and-reward"-Systeme (wie z. B. das Sammeln von Bonusmeilen) dienen der Steigerung von Nutzungsmotivation. Interaktive Werbeformen haben eine größere Nutzungschance und eine völlig andere Nutzungsqualität als herkömmliche Werbeformen. Nicht nur, dass sie sich nicht vermeiden lassen. Je mehr direkte Interaktion zwischen den Spielenden und dem Placement möglich ist, umso ausgeprägter ist das User-Engagement, also die aktive (kognitiv und affektive) und involvierte Werbenutzung - die ideale Bedingung für Werbewirkung.

Mit diesen technischen und inhaltlichen Entwicklungen im Medienbereich verändert sich auch das Werbeumfeld. Damit einher gehen neue Möglichkeiten der Werbetreibenden, aber auch angepassste Nutzungsformen der Rezipientinnen und Rezipienten. Zentrale aktuelle Entwicklungen sind hier sicherlich (wenn auch nicht ausschließlich) integrierte und hybride Werbeformen sowie die umfassende zeitliche und örtliche Nutzungssouveränität. Für die Werbenutzungsforschung ergibt sich daraus die Notwendigkeit, ihre Designs, Methoden und Messinstrumente anzupassen, um weiterhin reliabel und valide erheben zu können, wie und warum das Publikum Werbung nutzt bzw. sie meidet und welche Strategien sich eignen, um Werbenutzung in Werbewirkung zu überführen.

\section{Literatur}

Abernethy, A. M. (1991). Differences between advertising and program exposure for car radio listening. Journal of Advertising Reserach, 31(2), 33-42.

Alwitt, L. F. \& Prabhaker, P. R. (1992). Functional and belief dimensions of attitudes to television advertising. Implications for copytesting. Journal of Advertising Research, 32(5), 30-42.

Arbeitsgemeinschaft Fernsehforschung (o. J.). Forschung. Zugriff am 20.12.2013. Verfügbar unter http://www.agf.de/forschung/

Baek, T. H. \& Morimoto, M. (2012). Stay away from me: Examining the determinants of consumer avoidance of personalized advertising. Journal of Advertising, 41(1), 59-76.

Bauer, R. \& Greyser, S. A. (1968). Advertising in America: The consumer view. Bosten, MA: Harvard University.

Bellman, S., Schweda, A. \& Varan, D. (2010). The residual impact of avoided television advertising. Journal of Advertising, 39(1), 67-81.

Biocca, F. A. (1988). Opposing conceptions of the audience: The active and passive hemispheres of mass communication theory. In J. Anderson (Hrsg.), Communication Yearbook (Bd. 11, S. 51-80). New York, NY: Sage.

Blake, C., Schlütz, D. \& Herbst, B. (2012). Die Wahrnehmung von Online-Werbung: Eine Eye-Tracking-Studie. In H. Haas \& K. Lobinger (Hrsg.), Qualitäten der Werbung - Qualitäten der Werbeforschung (S. 265-282). Köln: von Halem.

Blumler, J. G. (1979). The role of theory in uses and gratifications studies. Communication Research, 6(1), 9-36.

Brehm, J. W. (1966). A theory of psychological reactance. New York, NY: Academic Press. 
Bucklin, L. P. (1965). The informative role of advertising. Journal of Advertising Research, 5(3), 6-10.

Burns, K. S. \& Lutz, R. S. (2006). The function of format: Consumer responses to six on-line advertising formats. Journal of Advertising, 35(1), 53-63.

Cho, C.-H. \& Cheon, H. J. (2004). Why do people avoid advertising on the internet? Journal of Advertising, 33(4), 89-97.

Cowley, E. \& Barron, C. (2008). When product placement goes wrong: The effects of program liking and placement prominence. Journal of Advertising, 37(1), 89-98.

Cronin, J. J. \& Menelly, N. E. (1992). Discrimination vs. avoidance: "Zipping" of television commercials. Journal of Advertising, 21(2), 1-7.

Danaher, P. J. (1995). What happens to television ratings during commercial breaks? Journal of Advertising Research, 35(1), 37-47.

Danaher, P. J. \& Mullarkey, G. W. (2003). Factors affecting online advertising recall: A study of students. Journal of Advertising Research, 43(3), 252-267.

Dardis, F. E., Schmierbach, M. \& Limperos, A. M. (2012). The impact of game customization and control mechanisms on recall of integral and peripheral brand placements in video games. Journal of Interactive Advertising 12(2), 1-12.

DeLorme, D. E. \& Reid, L., N. (1999). Moviegoers' experiences and interpretations of brands in films revisited. Journal of Advertising, 28(2), 71-94.

Diao, F. \& Sundar, S. S. (2004). Orienting response and memory for web advertisments: Exploring effects of pop-up window and animation. Communication Research, 31(5), 537-567.

Dix, S. \& Phau, I. (2010). Television advertising avoidance: Advancing research methodology. Journal of Promotion Management, 16(1-2), 114-133.

Downey, K. (2007). Study: First ad does get more eyeballs. That lead commercial holds onto far more viewers. Zugriff am 09.12.2013. Verfügbar unter http://www.medialifemagazine.com/study-first-ad-does-get-moreeyeballs/

Drèze, X. \& Hussherr, F.-X. (2003). Internet advertising: Is anybody watching? Journal of Interactive Marketing, 17(4), 8-23.

Ducofee, R. H. (1995). How consumers assess the value of advertising. Journal of Current Issues and Research in Advertising, 17(1), 1-18.

Ducofee, R. H. (1996). Advertising value and advertising on the web. Journal of Advertising Research, 36(5), 21-35.

Duff, B. R. L. \& Faber, R. J. (2011). Missing the mark: Advertising avoidance and distractor devaluation. Journal of Advertising, 40(2), 51-62.

Edwards, S. M., Li, H. \& Lee, J.-H. (2002). Forced exposure and psychological reactance: Antecedents and consequences of the perceived intrusiveness of pop-up ads. Journal of Advertising, 31(3), 83-95.

Ettenhuber, A. (2007). Die Beschleunigung des Fernsehverhaltens: Sekundäranalyse von Daten aus dem GfKFernsehpanel. München: R. Fischer.

Friestad, M. \& Wright, P. (1994). The persuasion knowledge model: How people cope with persuasion attempts. Journal of Consumer Research, 21(1), 1-31.

Friestad, M. \& Wright, P. (1995). Persuasion knowledge: Lay people's and resesarchers' beliefs about the psychology of advertising. Journal of Consumer Research, 22(1), 62-74.

Fulgoni, G. M. \& Mörn, M. P. (2009). Whither the click? How online advertising works. Journal of Advertising Research, 49(2), 134-142.

Gattringer, K. \& Klingler, W. (2013). Radionutzung auf hohem Niveau konstant. Media Perspektiven, o. Jg.(9), 441-453.

Gehrau, V. \& Schulze, A. (2013). Quantitative Beobachtung: Grundprinzipien und Anwendungen. In W. Möhring \& D. Schlütz (Hrsg.), Handbuch standardisierte Erhebungsmethoden in der Kommunikationswissenschaft (S. 329346). Wiesbaden: VS.

Greenberg, B. S. (1974). Gratifications of television viewing and their correlates for British children. In J. G. Blumler (Hrsg.), The uses of mass communications. Current perspectives on gratifications research (S. 71-92). Beverly Hills, CA: Sage.

Hammer, P., Riebe, E. \& Kennedy, R. (2009). How clutter affects advertising effectiveness. Journal of Advertising Research, 49(2), 159-163.

Heeter, C. \& Cohen, E. (1988). Viewing style differences between radio and television. In C. Heeter \& B. S. Greenberg (Hrsg.), Cableviewing (S. 113-122). Norwood, NJ: Ablex.

Heeter, C. \& Greenberg, B. S. (1985). Profiling the zappers. Journal of Advertising Research, 25(2) 15-19.

Hellmann, K.-U. (2006). Vorüberlegungen zur Funktion der Distinktion für den Konsum. In K.-S. Rehberg (Hrsg.), Soziale Ungleichheit, kulturelle Unterschiede (S. 395-406). Frankfurt/Main: Campus. Online verfügbar unter http://nbn-resolving.de/urn:nbn:de:0168-ssoar-145265 (Zugriff am 09.04.2014). 
Hofsümmer, K.-H. \& Müller, D. K. (1999). Zapping bei Werbung - ein überschätztes Phänomen. Eine Bestandsaufname des Zuschauerverhaltens vor und während der Fernsehwerbung. Media Perspektiven, o. Jg.(6), 296-300.

Katz, E., Blumler, J. G. \& Gurevitch, M. (1974). Utilization of mass communication by the individual. In J. G. Blumler (Hrsg.), The uses of mass communications. Current perspectives on gratifications research (S. 19-32). Beverly Hills, CA: Sage.

Kelly, L., Kerr, G. \& Drennan, J. (2010). Avoidance of advertising in social networking sites: The teenage perspecitive. Journal of Interactive Advertising, 10(2), 16-27.

Klimmt, C., Weinacht, S. \& Donner, B. (2009). Netzkompetenz gleich Werberesistenz? Ein Experiment zur Erinnerung von Online-Werbung in Abhängigkeit von der Interneterfahrung. Medienwirtschaft, 6(1), 6-17.

Kroeber-Riel, W., Weinberg, P. \& Gröppel-Klein, A. (2009). Konsumentenverhalten. Müchen: Vahlen.

Krugman, D. M., Cameron, G. T. \& McKearney White, C. (1995). Visual attention to programming and commercials: The use of in-home observations. Journal of Advertising, 24(1), 1-12.

Kuhlmann, C. \& Wolling, J. (2004). Fernsehen als Nebenbeimedium: Befragungsdaten und Tagebuchdaten im Vergleich. Medien \& Kommunikationswissenschaft, 52(3), 386-411.

Kwak, H., Andras, T. L. \& Zinkhan, G. M. (2009). Advertising to "active" viewers. Consumer attitudes in the US and South Korea. International Journal of Advertising, 28(1), 49-75.

LaRose, R. \& Eastin, M. S. (2004). A social cognitive theory of internet uses and gratifications: Towards a new model of media attendance. Journal of Broadcasting \& Electronic Media, 48(3), 358-377.

Lee, M. \& Faber, R. J. (2007). Effects of product placement in on-line games on brand memory: A perspective of the limited capacity model of attention. Journal of Advertising, 36(4), 75-90.

Levy, M. R. \& Windahl, S. (1985). The concept of audience activity. In K. E. Rosengren, L. A. Wenner \& P. Palmgreen (Hrsg.), Media gratifications research. Current perspectives (S. 109-122). Beverly Hills, CA: Sage.

Lewis, B. \& Porter, L. (2010). In-game advertising effects: Examining player perceptions of advertising schema congruity in a massively multiplayer online role-playing game. Journal of Interactive Advertising, 10(2), 46-60.

Li, H., Edwards, S. M. \& Lee, J.-H. (2002). Measuring the intrusiveness of advertisements: Scale development and validation. Journal of Advertising, 31(2), 37-47.

Matthes, J., Schemer, C. \& Wirth, W. (2007). More than meets the eye. Investigating the hidden impact of brand placements in television magazines. International Journal of Advertising, 26(4), 477-503.

McGuire, W. J. (1976). Some internal psychological factors influencing consumer choice. Journal of Consumer Research, 2(4), 302-319.

MacKenzie, S. B. \& Lutz, R. J. (1989). An empirical examination of the structural antecendents of attitude toward the ad in an advertising pretesting context. Journal of Marketing, 53(2), 48-65.

MacKenzie, S. B., Lutz, R. J. \& Belch, G. E. (1986). The role of attitude toward the ad as a mediator of advertising effectiveness: A test of competing explanations. Journal of Marketing Research, 23(2), 130-143.

Mitchell, V., Macklin, J. E. \& Paxmann, J. (2007). Social uses of advertising. An example of young male adults. International Journal of Advertising 26(2), 199-222.

Moorman, M., Neijens, P. \& Smit, E. G. (2007). The effects of program involvement on commercial exposure and recall in a naturalistic setting. Journal of Advertising, 36(1), 121-137.

Moriarty, S. E. \& Everett, S.-L. (1994). Commercial breaks: A viewing behavior study. Journalism \& Mass Communication Quarterly, 71(2), 346-355.

Naab, T. K. (2013). Gewohnheiten und Rituale der Fernsehnutzung. Theoretische Konzeption und methodische Perspektiven. Baden-Baden: Nomos.

Nelson, M. R. (2005). Exploring consumer response to „Advergaming“. In C. P. Haugtevedt, K. A. Machleit \& R. F. Yalch (Hrsg.), Online consumer psychology: Understanding and influencing consumer behaviour in the virtual world (S. 167-194). Mahwah, NJ: Lawrence Erlbaum Associates.

Nelson, M. R., Keum, H. \& Yaros, R. A. (2004). Advertainment or adcreep: Game players' attitudes toward advertising and product placements in computer games. Journal of Interactive Advertising, 5(1), 3-30.

Nicovich, S. G. (2005). The effect of involvement on ad judgment in a video game environment: The mediating role of presence. Journal of Interactive Advertising, 6(1), 29-39.

Nielsen. (2007). Nielsen launches commercial minute ratings in standardized file. Zugriff am 10.12.2013. Verfügbar unter http://www.nielsen.com/us/en/press-room/2007/Nielsen_Launches_Commercial_Minute _Ratings_in_Standardized_File.html

Obermiller, C. \& Spangenberg, E. R. (1998). Development of a scale to measure consumer scepticism toward advertising. Journal of Consumer Psychology, 7(2), 159-186.

O'Donohoe, S. (1994). Advertising uses and gratifications. European Journal of Marketing, 28(8/9), 52-75.

Ottler, S. (1998). Zapping: Zum selektiven Umgang mit Fernsehwerbung und dessen Bedeutung für die Vermarktung von Fernsehwerbezeit. München: R. Fischer. 
Pagendarm, M. \& Schaumburg, H. (2001). Why are users banner-blind? The impact of navigation style on the perception of web banners. Journal of Digital Information, 2(1). [Online]. Verfügbar unter http://journals.tdl.org/jodi/article/viewArticle/36/38 (21.06.2012).

Palmgren, P. \& Rayburn, J. (1982). Gratification sought and media exposure: an expectancy-value model. Communication Research, 9(4), 561-580.

Palmgreen, P. \& Rayburn, J. (1985). An expectancy-value approach to media gratifications. In K. E. Rosengren, L. A. Wenner \& P. Palmgreen (Hrsg.), Media gratifications research. Current perspectives (S. 61-72). Beverly Hills, CA: Sage.

Pasadeos, Y. (1990). Perceived informativeness of and irritation with local advertising. Journalism \& Mass Communication Quarterly, 67(1), 35-39.

Pavlou, P. A. \& Stewart, D. W. (2000). Measuring the effects and effectiveness of interactive advertising: A research agenda. Journal of Interactive Advertising, 1(1), 62-78.

Plummer, J. T. (1971). A theoretical view of advertising communication. Journal of Communication, 21(4), 315-325.

Pollay, R. W. \& Mittal, B. (1993). Here's the beef: Factors, determinants, and segments in consumer criticism of advertising. Journal of Marketing, 57(3), 99-114.

Ridder, C.-M. \& Hofsümmer, K.-H. (2001). Werbung in Deutschland - auch 2001 akzeptiert und anerkannt. Media Perspektiven, o. Jg.(6), 282-289.

Ridder, C.-M. \& Hofsümmer, K.-H. (2008). Wert und Relevanz der Werbung im öffentlich-rechtlichen Rundfunk. Media Perspektiven, o. Jg.(2), 50-57.

Rojaz-Méndez, J. \& Davis, G. (2005). Avoiding television advertising: Some explanations from time allocation theory. Journal of Advertising Research, 45(1), 34-48.

Rubin, A. M. (1984). Ritualized and instrumental television viewing. Journal of Communication, 34(3), 67-77.

Scherer, H. \& Schlütz, D. (2002). Gratifikation à la Minute: Die zeitnahe Erfassung von Gratifikationen. In P. Rössler, S. Kubisch \& V. Gehrau (Hrsg.), Empirische Perspektiven der Rezeptionsforschung (S. 133-151). München: R. Fischer.

Schlütz, D. \& Hartmann, L. (2012). Geminderter Nutzen: Reaktanz bei Facebook. In H. Scherer, D. Schlütz, H. Schmid-Petri \& A. Trommershausen (Hrsg.), Marken im Web 2.0: Theoretische Einordnung und empirische Erkenntnisse zur Markenkommunikation im Web 2.0 aus kommunikationswissenschaftlicher Sicht (S. 198-206). Köln: von Halem.

Schlütz, D., Blake, C., Charrier, D., Dyszy, A., Emde, K., Hirt, F., Keldenich, F., Link, E. \& Sponer, J. (2013). Werbung in Bildschirmspielen: Eine Eye-Tracking-Studie zur Rolle von Markenbekanntheit und Werbeintensität für die Wahrnehmung und Erinnerung von In-Game Advertising. In Thomas Schierl \& Jörg Tropp (Hrsg.), Wert und Werte der Marketing-Kommunikation (S. 281-298). Köln: von Halem.

Schlinger, M. J. (1979). A profile of responses to commercials. Journal of Advertising Research 19(2), 37-46.

Siddarth, S. \& Chattopadhyay, A. (1998). To zap or not to zap: A study of the determinants of channel switching during commercials. Marketing Science, 17(2), 124-138.

Siegert, G. \& Brecheis, D. (2010). Werbung in der Medien- und Informationsgesellschaft: Eine kommunikationswissenschaftliche Einführung (2. Aufl.). Wiesbaden: VS.

Simola, J., Kuisma, J., Öörni, A., Uusitalo, L. \& Hyönä, J. (2011). The impact of salient advertisements on reading and attention on Web page. Journal of Experimental Psychology: Applied, 17(2), 174-190.

Speck, P. S. \& Elliott, M. T. (1997). Predictors of advertising avoidance in print and broadcast media. Journal of Advertising, 26(3), 61-76.

Stipp, H. (2008). Werbung und Festplattenrekorder. Media Perspektiven, o. Jg.(6), 299-306.

Tan, S. J. \& Chia, L. (2007). Are we measuring the same attitude? Understanding media effects on attitude towards advertising. Marketing Theory, 7(4), 353-377.

TiVo. (2006). TiVo launches audience research and measurement (ARM) division. Zugriff am 10.12.2013. Verfügbar unter http://investor.tivo.com/phoenix.zhtml?c=106292\&p=irol-newsArticle\&ID= 1254259\&highlight $=$

Terlutter, R. \& Capella, M. L. (2013). The gamification of advertising: Analysis and research directions of in-game advertising, advergames, and advertising in social network games. Journal of Advertising, 42(2-3), 95-112.

Tsao, J. C. \& Sibley, S. D. (2004). Readership of free community papers as a source of advertising information: A uses and gratifications perspective. Journalism \& Mass Communication Quarterly, 81(4), 766-787.

Tse, A. C. B. \& Lee, R. P. W. (2001). Zapping behavior during commercial breaks. Journal of Advertising Research, 41(3), 25-29.

van Meurs, L. (1998). Zapp! A study on switching behavior during commercial breaks. Journal of Advertising Research, 38(1), 43-53.

Weber, P., Buchmann, S., \& Wirth, W. (2014). Schlechtes Ambiente? Die Bedeutung des Konsumenten-Involvements für die Wirkung moderat inkongruenter Ambient-Media. In H. Schramm \& J. Knoll (Hrsg.), Innovation der Persuasion. Die Qualität der Werbe- und Markenkommunikation in neuen Medienwelten (S. 200-217). Köln: Herbert von Halem. 
Wilbur, K. C. (2008). How the digital video recorder (DVR) changes traditional television advertising. Journal of Advertising, 37(1), 143-149.

Winkler, T. \& Buckner, K. (2006). Receptiveness of gamers to embedded brand messages in advergames: Attitudes toward product placement. Journal of Interactive Advertising, 7(1), 37-46.

Wirth, W. (2001). Aufmerksamkeit: Ein Konzept- und Theorieüberblick aus psychologischer Perspektive mit Implikationen für die Kommunikationswissenschaft. In K. Beck \& W. Schweiger (Hrsg.), Attention please: OnlineKommunikation und Aufmerksamkeit (S. 69-89). München: R. Fischer.

Wise, K., Bolls, P. D., Kim, H., Venkataraman, A. \& Meyer, R. (2008). Enjoyment of advergames and brand attitudes: The impact of thematic relevance. Journal of Interactive Advertising, 9(1), 27-36.

Wood, M., L. M., Nelson, M. R., Atkinson, L. \& Lane, J. B. (2008). Social utility theory: Guiding labeling of VNRs as ethical and effective public relations. Journal of Public Relations Research, 20(2), 231-249.

Yoo, C. Y., Kim, K. \& Stout, P. A. (2004). Assessing the effect of animation in online banner advertising: Hierarchy of effects model. Journal of Interactive Advertising, 4(2), 49-60.

Zanjani, S. H. A., Diamond, W. D. \& Chan, K. (2011). Does ad-context congruity help surfers and information seekers remember ads in cluttered e-magazines? Journal of Advertising, 40(4), 67-83.

Zajonc, R. B. (2001). Mere exposure: A gateway to the subliminal. Current Directions in Psychological Science, $10(6), 225-228$.

Zigmond, D. \& Stipp, H. (2010). Assessing a new advertising effect: Measurement of the impact of television commercials on internet search queries. Journal of Advertising Research, 50(2), 162-168.

Zufryden, F. S., Pedrick, J. H. \& Sankaralingam, A. (1993). Zapping and its impact on brand purchase behavior. Journal of Advertising Research, 33(1), 58-66. 